\title{
TP63 mutations are frequent in cutaneous melanoma, support UV etiology, but their role in melanomagenesis is unclear
}

\author{
PAOLA MONTI $^{1}$, PAOLA GHIORZO ${ }^{2,3}$, PAOLA MENICHINI ${ }^{1}$, GIORGIA FOGGETTI ${ }^{1}$, \\ PAOLA QUEIROLO $^{4}$, ALBERTO IZZOTTI ${ }^{1,5}$ and GILBERTO FRONZA ${ }^{1}$ \\ ${ }^{1}$ UOC Mutagenesis, Ospedale Policlinico San Martino; ${ }^{2}$ Department of Internal Medicine and Medical \\ Specialties, University of Genova; ${ }^{3}$ Genetics of Rare Cancers Unit and ${ }^{4}$ Medical Oncology Unit, Ospedale \\ Policlinico San Martino; ${ }^{5}$ Department of Health Sciences, University of Genova, I-16132 Genova, Italy
}

Received April 6, 2017; Accepted July 12, 2017

DOI: $10.3892 /$ or.2017.5903

\begin{abstract}
In contrast to TP53, cancer development is rarely associated with mutations in the TP63 and TP73 genes. Recently, next generation sequencing analysis revealed that TP63 mutations are frequent, specifically in cutaneous melanomas. Cutaneous melanoma represents $4 \%$ of skin cancers but it is responsible for $80 \%$ of skin cancer related deaths. In the present study, we first determined whether all three members of the P53 family of transcription factors were found mutated in cutaneous melanomas by retrieving all TP53, TP63 and TP73 mutations from cBioPortal (http://www. cbioportal.org/). TP53 and TP63 were frequently mutated [15.0\% (91/605) and 14.7\% (89/605), respectively], while TP73 [1.5\% (9/605)] was more rarely mutated ( $<<0.0001)$. A UV-mutation fingerprint was recognized for TP63 and TP73 genes. Then, we tried to evaluate the potential role of TP63 mutations as drivers or passengers in the tumorigenic process. In the former case, the amino acid substitutions should cause significant functional consequences on the main biochemical activity of the P63 protein, namely transactivation. The predicted effects of specific amino acid substitutions by two bioinformatics tools were rather different. Using a yeast-based functional assay, the observed hotspot mutant R379CP63 protein exhibited a substantial residual activity compared to the wild-type $(>70 \%)$. This result does not support a major role of the mutant P63 protein in melanomagenesis while it is still consistent with the TP63 gene being a recorder of UV exposure. The TP63 mutation spectrum from cutaneous melanomas, when compared with that observed at the germinal level in patients affected by P63-associated diseases [ectodermal dysplasia syndromes, (EDs)], revealed significant differences. The TP63 mutations were more frequent at
\end{abstract}

Correspondence to: Dr Gilberto Fronza, UOC Mutagenesis, Ospedale Policlinico San Martino, I-16132 Genova, Italy

E-mail: gilberto.fronza@hsanmartino.it

Key words: TP63, mutations, cutaneous melanomas, functional analysis, yeast assay
CpGs sites $(\mathrm{p}<0.0001)$ in EDs and at PyPy sites $(\mathrm{p}<0.0001)$ in cutaneous melanomas. The two spectra differed significantly $(\mathrm{p}<0.0001)$. We conclude that TP63 mutations are frequent in cutaneous melanoma, support UV etiology, but their role in melanomagenesis is unclear.

\section{Introduction}

The P53 family of tetrameric transcription factors is composed of the P53, P63 and P73 proteins (1-3) that influence many cellular pathways including cell proliferation, apoptosis, DNA repair, angiogenesis, metabolism and differentiation (4-6). All three proteins share an N-terminal transactivation domain, a central sequence-specific DNA-binding domain and a C-terminal oligomerization domain. Only P63 and P73 proteins contain an additional sterile- $\alpha$-motif domain within the C-terminus. Moreover, the P53 family members can be expressed as different isoforms both at the N-terminus $(\Delta \mathrm{N}$ and TA) and at the C-terminus ( $\alpha-\gamma$ for P53; $\alpha-\varepsilon$ for P63; $\alpha-\eta$ for P73) through the usage of two alternative promoters and alternative splicing mechanisms, respectively.

Despite protein and gene structure similarities, the overlap in cellular functions between P53, P63 and P73 is limited as highlighted by comparing their role in human physiology. P53 is a well-known tumor suppressor protein encoded by one of the most frequently mutated genes in sporadic types of cancer. Furthermore, TP53 germline mutations are associated with the development of the cancer prone Li-Fraumeni and Li-Fraumeni like syndromes (7-9). Conversely, P63 is critical for the correct development of ectodermal-derived tissues, whereas P73 contributes to neural and immune system functions $(10,11)$. No genetic disorder has been linked to TP73 germinal mutations (12), whereas heterozygous mutations in the TP63 gene underlie a subset of human ectodermal dysplasia syndromes (EDs) $(13,14)$.

Despite the fact that cancer development is rarely associated with TP63 and TP73 mutations, both genes play an active role in carcinogenesis. Flores et al (15) demonstrated the contribution of $\mathrm{P} 63$ and $\mathrm{P} 73$ in tumor development by revealing that $\mathrm{TP}^{\mathrm{H}} \mathrm{3}^{+-}$and $\mathrm{TP} 73^{+/-}$mice develop malignant tumors at a higher frequency with respect to the wild-type counterparts. In addition, $T P 53^{+/-} \mathrm{TP} 63^{+/-}$and $T P 53^{+/-} \mathrm{TP} 73^{+/-}$compound 
mutant mice developed a more severe phenotype (e.g., more metastases) compared to the $\mathrm{TP}^{\mathrm{H} 3^{+-}}$mice, indicating that P63 and P73 have functions independent of P53.

The tumor-suppressive activities of P63 and P73 appear to be tissue-specific. In fact, the tumor spectrum detected in mice defective for TP63 and TP73 is quite different from that of TP53-deficient mice (16). $T P 63^{+/-}$and $T P 73^{+/-}$mice develop primarily lung adenomas $\left(T P 63^{+/-}, 25 \% ; \mathrm{TP}^{++/}, 40 \%\right)$ squamous cell hyperplasia $\left(\mathrm{TP}^{+1 /-}, 50 \% ; \mathrm{TP}^{+/-}, 30 \%\right)$, while $T P 53^{+-}$mice primarily develop thymic lymphomas and osteosarcomas. These differences are probably due to the different patterns of expression of the P53 family members, while P53 is ubiquitous, the P63 and P73 proteins are highly expressed in epithelial tissues.

The observation that mutagens are carcinogens and that different mutagens induce specific spectra of mutations in genes frequently mutated in cancer (17), suggested that the analysis of the site and nature of DNA changes in genes mutated in tumors could be useful at identifying the factors contributing to the development of a specific tumor type $(18,19)$. Molecular epidemiology converged to the TP53 gene, positioning it at the crossroad of molecular carcinogenesis and risk assessment (18). This was due to the fact that the TP53 gene: i) is very sensitive to point mutations; ii) alterations in its coding sequence occur in $>50 \%$ of all human types of cancer; and iii) $\sim 80 \%$ of these alterations are missense mutations leading to proteins with a single amino acid substitution (20). The relationship between the exposure to a carcinogen and the presence of specific TP53 mutations in specific tumors has been extensively studied and described (21). TP53 mutations identified in tumors and cell lines have been collected in a database that contains more than 30,000 entries (http://p53.fr/) (20).

With regard to sunlight, the International Agency for Research on Cancer in 1992 classified UV radiation as a human carcinogen (22). Upon reaching the skin, UV photons are absorbed by electrons of DNA, leading to the formation between adjacent pyrimidines of photoproducts known as cyclobutane pyrimidine dimers (CPDs) and pyrimidine 6-4 pyrimidone photoproducts (6-4PPs) (23). Incorrect DNA repair leads to fixation of specific $\mathrm{C} \rightarrow \mathrm{T}$ and $\mathrm{CC} \rightarrow \mathrm{TT}$ transitions at di-pyrimidine sites (24). About $50 \%$ of skin cancers exhibit the signature of UV-induced mutagenesis on TP53 (18). TP53 mutations are frequent in both normal-appearing sun-exposed skin and premalignant actinic keratosis lesions, which are considered precursors of squamous cell carcinoma.

The introduction of the next generation sequencing (NGS) approaches on cancer cell genomes to determine the whole landscape of mutations in cancers, profoundly changed the perspective of mutational fingerprints. While the Sanger sequencing imposed a technical threshold (a mutation is detectable in a single sample if it is present in $\sim 15-20 \%$ of the alleles), NGS can detect with accuracy hundreds of mutations in a single sample even if it is present in 1\% (and even less) of the alleles.

The identification of gene mutations that directly or indirectly confer a selective growth advantage to the cell in which they occur ('driver mutations') among a plethora of other mutations devoid of a role in the cancer process ('passenger mutations') is a formidable challenge. The situation is even more complicated, since a driver gene (i.e., that can harbor driver mutations) may also contain passenger mutations. A review suggests that, considering all types of tumors, the total number of driver genes in cancer which was discovered through the NGS approach is $\sim 125,29 \%$ of which was discovered through the NGS approach (25). Numerous statistical methods have been described to distinguish between driver versus passenger mutations (26-28). Furthermore, the number of driver mutations sufficient for a cell to evolve into an advanced cancer is thought to be extremely low. In solid tumors of adults, including melanoma, alterations in as few as three driver genes are sufficient $(29,30)$. This pointed towards the idea that pathways, rather than individual genes, appear to govern the tumorigenic process (25), which has rendered complex cancergenome landscapes intelligible and exploitable.

Cutaneous melanoma represents $4 \%$ of skin cancers but is responsible for $80 \%$ of skin cancer related deaths, corresponding to $\sim 1-2 \%$ of all cancer related deaths (31). Cutaneous melanoma, which develops from epidermal melanocytes of the skin, represents the most common melanoma. Sun exposure is accepted as a major causative factor of melanomas (32). Cutaneous melanomas have been studied by NGS since 2010 (33). This analysis generated the first comprehensive catalogue of somatic mutations from a malignant melanoma, revealing that the predominant mutational signature reflects DNA damage due to UV exposure. Moreover, through analysis of large-scale melanoma exome data, Hodis et al (34) discovered six novel driver melanoma genes (PPP6C, RAC1, SNX31, TACC1, STK19 and ARID2), providing unequivocal genomic evidence for a direct mutagenic role of UV light in melanoma pathogenesis. Recently, the sequence of genetic alterations during melanoma progression was defined, revealing distinct evolutionary paths for different melanoma subtypes $(35,36)$. While benign lesions harbored BRAF V600E mutations exclusively, those classified as 'intermediate' were enriched for NRAS mutations and additional driver mutations. TERT promoter mutations were observed at a high incidence in intermediate lesions and melanomas in situ. Biallelic CDKN2A inactivation emerged exclusively in invasive melanomas, while PTEN and TP53 mutations were found only in advanced primary melanomas and their presence appears to represent the transition to metastatic tumors (36).

Recently, using NGS, missense mutations in the TP63 gene were frequently and specifically found in cutaneous melanomas ( 15\%) (37). This observation prompted us to study the features of TP63 mutations from the molecular epidemiology point of view and to ascertain whether all three members of the P53 family were mutated in melanomas with equal frequency.

\section{Materials and methods}

Data collection. A total of 605 cases were considered by reviewing all studies documenting TP53/TP63/TP73 mutations in cutaneous melanomas retrieved from cBioPortal (http:// www.cbioportal.org) $(38,39)$. Eighty nine of them exhibited a total of 111 TP63 mutations, 91 cases exhibited a total of 105 TP53 mutations and only 9 cases exhibited a total of 10 TP73 mutations $(40,41)$.

TP63 mutations associated to EDs were retrieved from literature reporting amino acid substitutions and the causative single base pair substitution deduced from the genetic 
code $(13,42,43)$. Excluding a tandem mutation (CGC $>$ CAA R318Q), a total of 101 TP63 base pair substitutions found in EDs were considered.

In order to infer the potential role of the different mutations in terms of driver or passenger mutations, the output of two bioinformatic predictors, namely mutation assessor and functional analysis through hidden Markov models, FATHMM-MKL (an algorithm which predicts the functional, molecular and phenotypic consequences of protein missense variants using hidden Markov models, and classifies as 'pathogenic' or 'neutral' mutations if the scores are $\geq 0.7$ or $\leq 0.5$, respectively) were retrieved from cBioPortal.

Statistical analysis. In order to rigorously compare mutational spectra observed at the same locus, i.e., considering where along the nucleotide sequence the mutation was observed, and how many independent mutations were observed in each specific position, the Adams and Skopek algorithm (44) and the program from Cariello et al were used (45).

The Adams and Skopek algorithm uses a Monte Carlo method to simulate a P-value of the standard hypergeometric test for a contingency table (44). Unlike the $\chi^{2}$ test, which can also be applied to contingency tables and requires that all cells contain five or more events, the hypergeometric test is appropriate when applied to sparse data sets, often found in mutational spectra analyses. The Cariello et al program (45) uses a random number generator to produce a large number of simulated spectra based on the hypergeometric probability of the experimentally observed input spectra. The degree to which the simulated spectra differ from the input spectra is used to estimate the probability that the two input spectra were derived from the same population. A P-value $<0.05$ leads to the rejection of the null hypothesis and to the conclusion that the input spectra are different.

Yeast strains and media. The issue of P53 family transactivation specificity was thoroughly investigated by our group taking advantage of the previously described yeast functional assay (luciferase reporter-based assay) $(8,9,20,46)$. In particular, in order to evaluate the functionality of the P63 R379C allele, it was cloned into a yeast expression vector as $\Delta \mathrm{N}-\mathrm{P} 63 \alpha$ and TA-P63 $\alpha$ isoforms; their functionality was evaluated in two yeast reporter strains containing the luciferase gene under the control of the P63 responsive genes P21 and PERP. yLFM-P21-5' and yLFM-PERP yeast strains were used (14); the two strains are isogenic except for the different Response Element (RE) (20 bp) located upstream the luciferase reporter gene. Cells were grown in YPDA medium (1\% yeast extract, $2 \%$ peptone, $2 \%$ dextrose, $200 \mathrm{mg} / \mathrm{l}$ adenine) or in a selective medium containing dextrose or raffinose as a carbon source and adenine $(200 \mathrm{mg} / \mathrm{l})$ but lacking tryptophan (SigmaAldrich, St. Louis, MO, USA; BiokarDiagnostics, Pantin, France). Galactose was added to the medium to modulate P63 expression under the inducible GAL1-GAL10 promoter.

Yeast vectors. pTSG-based vectors expressing wild-type $\Delta \mathrm{N}-\mathrm{P} 63 \alpha$ and TA-P63 $\alpha$ isoforms were already available (14). Yeast vectors expressing mutant isoforms $(\Delta \mathrm{N}-\mathrm{P} 63 \alpha \mathrm{R} 379 \mathrm{C}$ and TA-P63 $\alpha$ R379C) were constructed using the experimental strategy as previously described $(9,14)$. Briefly, for R379C mutation, a pair of complementary oligonucleotides (which served as forward and reverse primers) was synthesized, with the mutated nucleotide (underlined the corresponding codon) adjacent to the central position (R379C forward, 5'-aagcgccegt tttgtcagaacacacatggt-3' and reverse, 5 '-accatgtgtgttctgacaaaacgggcgctt- 3 '). Forward and reverse primers, paired respectively with $\alpha$-Cter and $\Delta \mathrm{N}$-Nter (or TA-Nter) primers $(\Delta \mathrm{N}-\mathrm{Nter}$, 5 -caagctataccaagcatacaatcaactatctcatatacagttaactcgagatgttgta cctggaaaacaat-3'; TA-Nter, 5'-caagctataccaagcatacaatcaactatctcatatacagttaactcgagatgtcccagagcacacagaca-3'), were used in two separate PCR reactions with the pcDNA3.1 TP63 $\alpha$ plasmid ( $\triangle \mathrm{N}$ or TA isoform) as template. The following PCR conditions were used: $1 \mathrm{~min}$ of denaturation at $94^{\circ} \mathrm{C}, 1 \mathrm{~min}$ of annealing at $55^{\circ} \mathrm{C}$ and $2 \mathrm{~min}$ of elongation at $72^{\circ} \mathrm{C}$ (35 cycles) (Exact polymerase, 5 prime). Unpurified aliquots of PCR products were then transformed in yIG397 yeast strain together with an XhoI/NotI (New England Biolabs, Ipswich, MA, USA) digested pTS-based vector. In yeast, the plasmid is re-sealed together with the PCR products by recombination, exploiting the sequence homology at the end of the fragments (gap repair assay) $(8,9)$. Plasmid DNA was recovered from yeast colonies, expanded in E. coli, and checked by digestion; the presence of the specific mutation was verified by DNA sequencing (BMR Genomics, Padova, Italy).

Yeast vectors expressing R379C mutant protein $(\Delta \mathrm{N}-\mathrm{P} 63 \alpha$ and TA-P63 $\alpha$ isoforms) under the inducible GAL1-GAL10 promoter (pTSG-based), were constructed by double-digesting the pTS-based expression vectors with the XhoI and NotI restriction enzymes. The DNA fragment containing the mutant $\Delta \mathrm{N}$ - or the TA-TP63 $\alpha$ coding sequence (cDNA) was then cloned in a double-digested pTSG-based vector (14).

Yeast functional assay. P63 wild-type and mutant isoforms $(\Delta \mathrm{N}-\mathrm{P} 63 \alpha$ and TA-P63 $\alpha$ ) were expressed by a pTSG-based vector (TRP1 selection marker) (14). Plasmid pRS314 (TRP1) was used as an empty vector. The functional assay was conducted according to the miniaturized protocol we developed $(14,46)$. Briefly, yeast strains were transformed (LiAc method) with the pTSG-based expression vectors along with the empty vector. Yeast transformants were resuspended in minimal synthetic medium containing raffinose plus galactose $(0,128 \%)$ in a final volume of $200 \mu \mathrm{l}$ (96-well plates) and were grown for $8 \mathrm{~h}$ with vigorous shaking at $30^{\circ} \mathrm{C}$. The OD $(600 \mathrm{~nm})$ was then directly assessed in the transparent 96-well plate. Twenty microliters of cell suspension were transferred in a white plate and mixed with an equal volume of PLB buffer 2X (Passive Lysis Buffer; Promega, Madison, WI, USA) in order to obtain the lysis of yeast cells. After $15 \mathrm{~min}$ of shaking at room temperature, $20 \mu \mathrm{l}$ of Firefly luciferase substrate (Bright Glo; Promega) were added. The luciferase activity was assessed using a multilabel plate reader (Mithras LB 940; Berthold Technologies, Bad Wildbad, Germany) and normalized to OD $600 \mathrm{~nm}$. The transactivation ability of the wild-type and mutant P63 proteins was expressed as fold of induction over empty vector for each reporter strain; the percentage of the luciferase activity of the P63 mutants with respect to the wild-type was also calculated.

Western blot analysis. Yeast transformants in the yLFM-P21-5' and yLFM-PERP strains were grown $8 \mathrm{~h}$ in selective medium 
Table I. Comparison of TP53, TP63 and TP73 mutations in cutaneous melanomas (n=605).

\begin{tabular}{lccc}
\hline Types of mutations & $T P 53$ & $T P 63$ & $T P 73$ \\
\hline No. of cases with mutations & 91 & 89 & 9 \\
No. of cases with mutations/total (\%) & $91 / 605(15.0)^{\mathrm{a}}$ & $89 / 605(14.7)^{\mathrm{b}}$ & $9 / 605(1.5)^{\mathrm{a}, \mathrm{b}}$ \\
No. of mutations & 105 & 111 & 10 \\
Type of mutations & & & 0 \\
Frameshift Del/Ins & 8 & 1 & 0 \\
Tandem mutations & 2 & 1 & 0 \\
Splice mutations & 7 & 8 & 10 \\
Single bp & 88 & 101 & $1(10)$ \\
Classes of bp, n (\%) & & & $1(10)$ \\
AT>CG & $5(6)$ & $0(0)$ & $1(10)$ \\
AT>GC & $6(7)$ & $4(4)$ & $5(50)$ \\
AT $>$ TA & $5(6)$ & $3(3)$ & $2(20)$ \\
GC $>$ AT & $65(73)$ & $92(91)$ & $0(0)$ \\
GC $>$ CG & $5(6)$ & $1(1)$ & $1 / 10(10)$ \\
GC $>$ TA & $2(2)$ & $1(1)$ & $8 / 10(80)$ \\
Localization at CpGs, n/total (\%) & $23 / 88(26)$ & $37 / 101(37)$ & $98 / 101(97)$ \\
Localization at PyPy, n/total (\%) & $75 / 88(85)$ & & \\
\end{tabular}

Data from cBioPortal for Cancer Genomics for cutaneous melanomas. ${ }^{\mathrm{a}} \mathrm{p}<0.0001,{ }^{\mathrm{b}} \mathrm{p}<0.0001$. PyPy, pyrimidines.

containing $0.128 \%$ galactose and collected by centrifugation. Cells were resuspended in $100 \mu 1$ distilled water, and $100 \mu 1$ $0.2 \mathrm{M} \mathrm{NaOH}$ were then added. The mixture was then incubated for $5 \mathrm{~min}$ at room temperature, pelleted, resuspended in $50 \mu \mathrm{l}$ SDS sample buffer (0.06 M Tris- $\mathrm{HCl}, \mathrm{pH} 6.8,5 \%$ glycerol, $2 \%$ SDS, $4 \% \beta$-mercaptoethanol, $0.0025 \%$ bromophenol blue), boiled for $3 \mathrm{~min}$ and pelleted again (47). Ten microliters of yeast supernatant were resolved on 4-15\% Mini-Protean TGX gels and transferred to nitrocellulose membranes by Trans-Blot Turbo Blotting System using the Trans-Blot Transfer Pack, (Bio-Rad, Hercules, CA, USA). A specific antibody directed against P63 (clone 4A4, sc-8431; Santa Cruz Biotechnology, Santa Cruz, CA, USA) was diluted in $1 \%$ non-fat dry milk dissolved in PBS-T. Phosphoglycerate kinase 1 (PGK1) was used as a loading control (clone 22C5D8; Life Technologies; Thermo Fisher Scientific, Inc., Waltham, MA, USA). After washing, the blots were incubated with the appropriate IgG horseradish peroxidase-conjugated secondary antibody (antimouse IgG HRP, A9044; Sigma-Aldrich, St. Louis, MO, USA), and immune complexes were visualized with ECL Fast Pico (Immunological Sciences, Rome, Italy). Chemiluminescence was analyzed by Alliance LD (Uvitec, Cambridge, UK).

\section{Results}

TP53 and TP63, but not TP73, are frequently mutated in cutaneous melanomas. All TP53, TP63 and TP73 mutations in cutaneous melanomas were retrieved from the cBioPortal (http://www.cbioportal.org) $(38,39)$. Overall, the incidence of TP53 and TP63 mutations was similar $[15.0 \%$ (91/605) and 14.7\% (89/605) for TP53 and TP63, respectively] while the frequency of $T P 73$ mutations [1.5\% (9/605)] was significantly lower $(\mathrm{p}<0.0001)$. Since some melanomas exhibited more than a single mutation, a total of 105 TP53, 111 TP63 and 10 TP73 mutations were retrieved and their molecular features analyzed (Table I). Clearly, for all three genes the majority $(>83 \%)$ of mutations were single base pair substitutions $($ TP53, 88/105=83.8\%; TP63, 101/111=91\%; $T P 73,10 / 10=100 \%$ ), at least $80 \%$ of which were localized at adjacent pyrimidines (PyPy). At least $50 \%$ of all base pairs substitutions for all three genes were GC $>$ AT transitions. Their codon localization along each cDNA sequence is represented in Fig. 1.

Thus, TP53 and TP63, but not TP73, mutations are frequently found in cutaneous melanomas and their molecular features are reminiscent of a UV-exposure, suggesting that TP53 and TP63 genes behave like two 'recorders of UV-exposure'. This conclusion is indirectly supported by the fact that among 80 patients with uveal melanomas, a form of melanoma that should be less UV-dependent since the UV radiation should not have the power to hit the target tissue, none of them displayed TP53 and TP63 mutations (TCGA study) (http://www.cbioportal.org).

The TP63 mutation fingerprint in cutaneous melanomas is distinguishable from the one observed in P63-associated $E D s$. To verify the assumption of molecular epidemiology, namely that carcinogens leave distinguishable fingerprints, 101 germinal TP63 mutations found in patients affected by a specific subset of EDs were retrieved from the literature $(13,42,43)$. Excluding a tandem mutation (1 C $\underline{\text { GC }}>$ CAA, R318Q) with no feature of UV-induced 

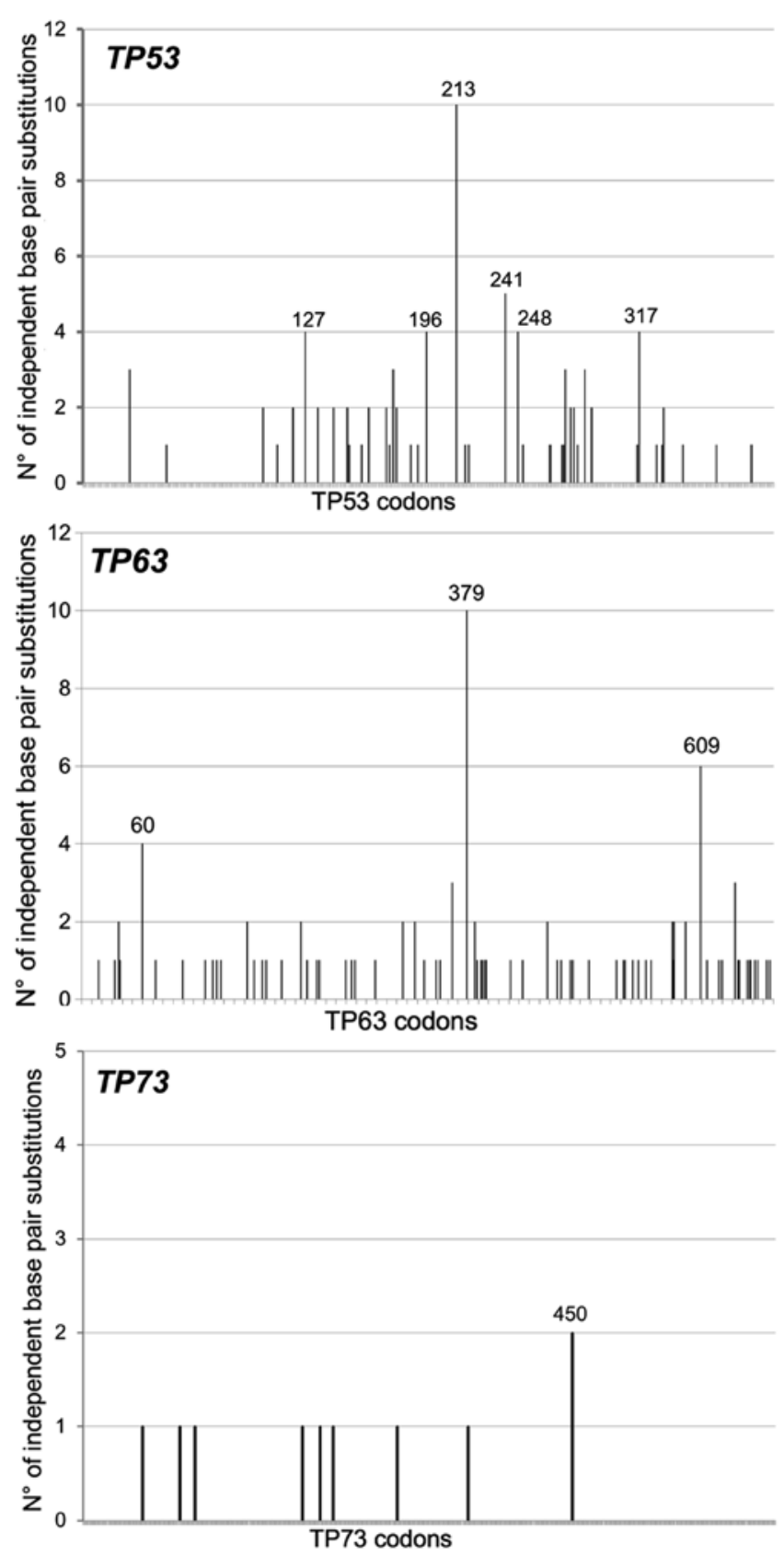

Figure 1. Sequence distribution along the TP53, TP63 and TP73 nucleotide positions of independent single base pair substitutions in cutaneous melanomas retrieved from cBioPortal (http://www.cbioportal.org).

tandem mutation, a total of 100 TP63 base pair substitutions were analyzed and compared with the TP63 mutations found in cutaneous melanomas (Table II). The genesis of the former mutations is most likely unrelated to UV-exposure. If the classes of mutations were considered, the only significant difference between the two spectra is a higher percentage of GC>AT transitions in melanomas than in EDs $(92 / 101$; $91 \%$ vs. 76/101; 76\%; p<0.0005). However, significant and interesting differences emerged when the localization of mutations at $\mathrm{CpGs}$ or PyPy sites was considered. Indeed, significantly more TP63 mutations were located at CpGs in EDs than in melanomas (74 vs. 37\%; p<0.0001), and vice versa, significantly more TP63 mutations were located at PyPy in melanomas than in EDs (97 vs. 70\%;

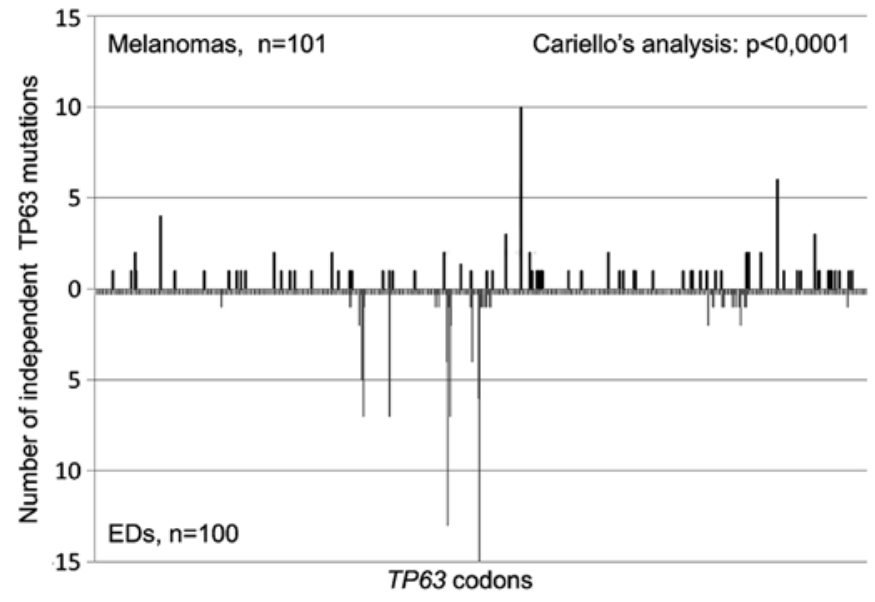

Figure 2. Distribution of TP63 single base pair substitutions found in cutaneous melanomas $(n=101)$ and at the germinal level in patients affected by P63-associated diseases (EDs). The two mutation spectra compared using the Cariello et al program (45) differed significantly $(\mathrm{p}<0,0001)$. EDs, ectodermal dysplasia syndromes.

p<0.0001). Finally, the two TP63 mutation spectra appeared significantly different $(\mathrm{p}<0.0001$; Fig. 2) also when the sequence distribution of independent base pair substitutions along the TP63 nucleotide sequence was compared using the Cariello et al program (45), which provides a rigorous statistical test of the relatedness of two spectra at the same locus.

In conclusion, using a mutational spectrometry approach we demonstrated that a UV fingerprint could be recognized in the TP63 mutations found in cutaneous melanomas and that such a mutation spectrum is distinguishable from the one observed in EDs.

TP53 and TP63 mutations in cutaneous melanomas: drivers, or passengers? A tentative answer from bioinformatics prediction of the functional consequences of TP53 and TP63 mutations. Since TP53 and TP63 mutations are relatively frequent in cutaneous melanomas a question arises. Do these mutations play a role as potential drivers or passengers in the tumorigenic process? To answer this question, for each mutation we first registered the classifications (Mutation Assessor and FATHMM-MKL) reported at the cBioPortal (http://www. cbioportal.org) $(41,42)$. To act as a driver, the mutations should have consequences on the functionality of the P53/P63 proteins (i.e., amino acid substitution should cause a change in transactivation capacity of the mutant with respect to the wild-type protein). When the functional consequences of mutations were evaluated using the Mutation Assessor bioinformatics tool a significantly larger proportion of TP53 mutations (TP53: 60/64, 93.7\%; TP63: 32/95, 33.7\%; p<0.0001, Fisher's exact test) were classified to have a medium impact on protein functionality, while a significantly larger proportion of TP63 mutations (TP63: 42/95, 44.2\%; TP53: 2/64, 3.1\%; p<0.0001 Fisher's exact test), were classified to have a neutral impact on protein functionality (Table III). When prediction was performed with the FATHMM-MKL bioinformatics tool each and every single amino acid substitution in TP63 was predicted to be pathogenic (score>0.89) (Table III). Furthermore, no significant difference emerged among specific mutations since almost 
Table II. Comparison of TP63 mutations in cutaneous melanomas and in a subset of human EDs.

\begin{tabular}{|c|c|c|c|}
\hline Type of mutations & Melanomas $^{\mathrm{a}}$ & $\mathrm{EDs}^{\mathrm{b}}$ & $\begin{array}{c}\text { Melanoma vs. ED } \\
\text { (P-value) }\end{array}$ \\
\hline No. of mutations & 111 & 101 & \\
\hline \multicolumn{4}{|l|}{ Type of mutations } \\
\hline Frameshift Del/Ins & 1 & 0 & \\
\hline Tandem mutations & 1 & 1 & \\
\hline Splice mutations & 8 & 0 & \\
\hline Single bp substitutions & 101 & 100 & \\
\hline \multicolumn{4}{|l|}{ Classes of bp substitutions, $\mathrm{n}(\%)$} \\
\hline $\mathrm{AT}>\mathrm{CG}$ & $0(0)$ & $4(4)$ & NS \\
\hline $\mathrm{AT}>\mathrm{GC}$ & $4(4)$ & $9(9)$ & NS \\
\hline $\mathrm{AT}>\mathrm{TA}$ & $3(3)$ & $3(3)$ & NS \\
\hline $\mathrm{GC}>\mathrm{AT}$ & $92(91)$ & $76(76)$ & $<0.0005$ \\
\hline $\mathrm{GC}>\mathrm{CG}$ & $1(1)$ & $3(3)$ & NS \\
\hline $\mathrm{GC}>\mathrm{TA}$ & $1(1)$ & $5(5)$ & NS \\
\hline Localization at CpGs, n/total (\%) & 37/101 (37) & $74 / 100(74)$ & $<0.0001$ \\
\hline Localization at PyPy, n/total (\%) & 98/101 (97) & $70 / 100(70)$ & $<0.0001$ \\
\hline
\end{tabular}

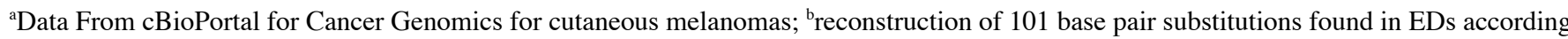
to literature. EDs, ectodermal dysplasia syndromes; PyPy, pyrimidines; NS, not significant.

Table III. Functional evaluation of amino acid substitutions using bioinformatics tools.

\begin{tabular}{|c|c|c|c|c|}
\hline Type of mutations & TP53 & TP63 & $T P 73$ & $\begin{array}{c}\text { TP53 vs. TP63 } \\
\text { (P-value) }\end{array}$ \\
\hline No. of mutations & 105 & 111 & 10 & \\
\hline Single bps & 88 & 101 & 10 & \\
\hline \multicolumn{5}{|l|}{$\begin{array}{l}\text { Missense mutations with } \\
\text { predicted functional } \\
\text { consequences } \\
\text { according to: }\end{array}$} \\
\hline Mutation Assessor & 64 & 95 & 9 & \\
\hline Neutral & 2 & 42 & 6 & $<0.0001$ \\
\hline Low & 2 & 21 & 1 & $<0.009$ \\
\hline Medium & 60 & 32 & 2 & $<0.0001$ \\
\hline High & 0 & 0 & 0 & \\
\hline FATHMM prediction & 88 & 79 & 7 & \\
\hline Neutral & 2 & 0 & 3 & NS \\
\hline Pathogenic & 86 & 79 & 4 & NS \\
\hline
\end{tabular}

Since the numerosity of the TP73 group is one order of magnitude lower than those of the TP63 and TP53 samples, only statistical comparisons performed between the TP53 vs. TP63 group of samples are reported. NS, not significant.

all TP53 and TP63 mutations were classified as pathogenic. Therefore, independent bioinformatics tools reveal a role of TP53 as a driver gene in cutaneous melanomas, while for TP63 mutations the situation is less clear. However, the abundance of TP63 mutations (similar to the one of TP53) along with the FATHMM-MKL prediction reveal that TP63 mutations may play some role in cutaneous melanomas.

From bioinformatics prediction to experimental determination of P63 functionality: the P63 R379C hotspot mutant protein in melanomas retains residual wild-type function. In order to shed more light on the functional effects of amino acid substitutions, we experimentally determined the outcome of the substitution of the arginine at codon 379 with a cysteine (R379C) on the transactivation ability of the P63 protein. This mutant protein represents the most frequent amino acid substitution observed in cutaneous melanomas (mutation hotspot). Furthermore, while Mutation Assessor predicts that this amino acid substitution will have a low impact on functionality, conversely FATHMM predicts it as pathogenic. For this purpose the P63 R379C allele was cloned into a yeast expression vector as $\Delta \mathrm{N}-\mathrm{P} 63 \alpha$ and TA-P63 $\alpha$ isoforms, and their functionality was evaluated in two yeast reporter strains containing the luciferase gene under the control of the P63 responsive genes P21 and PERP (see materials and methods for details).

The results revealed that the $\mathrm{R} 379 \mathrm{C}$ amino acid substitution did not markedly affect the transactivation ability of $\triangle \mathrm{N}-\mathrm{P} 63 \alpha$ and TA-P63 $\alpha$ proteins with respect to the wild-type (Fig. 3A). Specifically, when expressed as the $\Delta \mathrm{N}-\mathrm{P} 63 \alpha$ isoform the R379C protein exhibited a transactivation activity comparable to that of the wild-type P63. When expressed as the TA-P63a isoform, the same amino acid substitution exhibited, in both yeast strains, a decrease of transactivation ability of $\sim 30 \%$ with respect to the wild-type. Such a difference in transactivation 

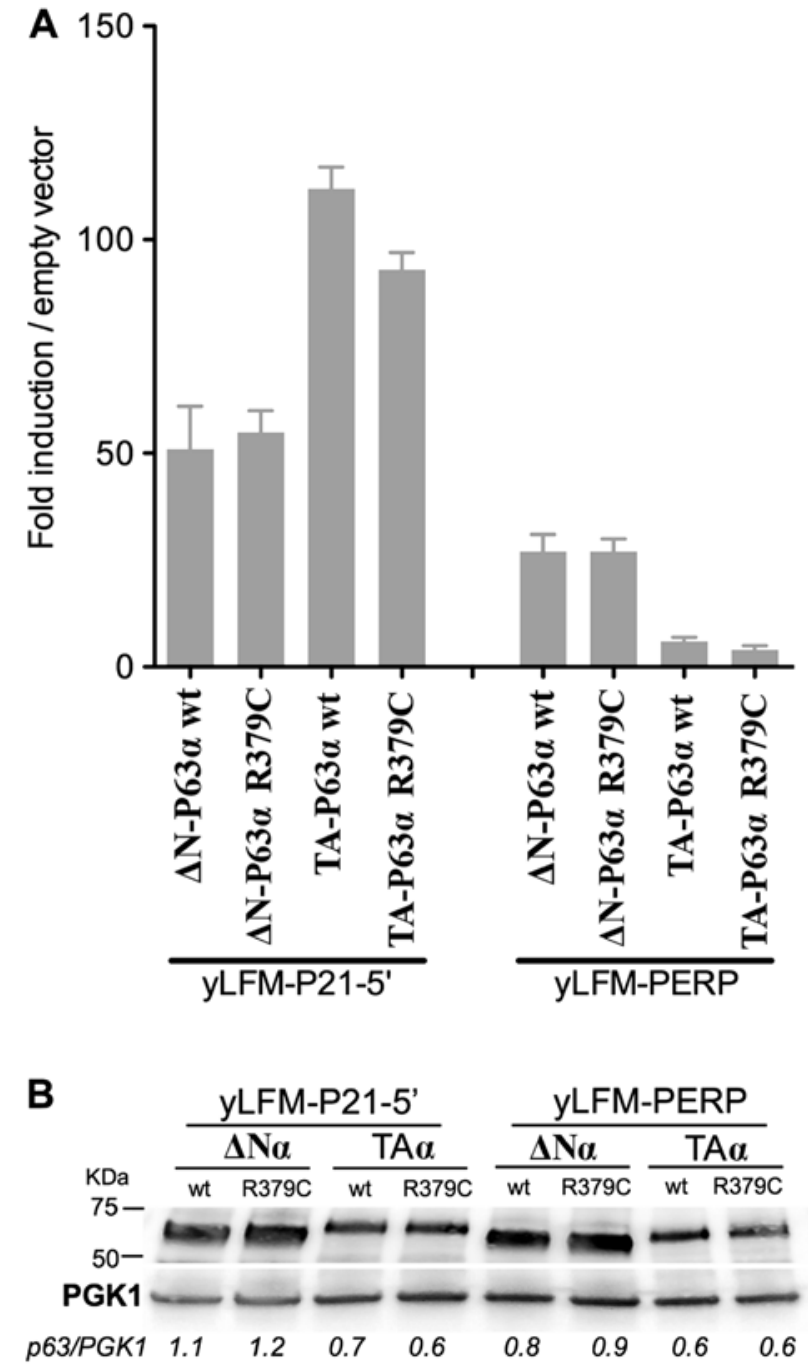

Figure 3. (A) Functional characterization of the mutant R379C P63 protein using the yeast-based transactivation assay. $\Delta \mathrm{N}-\mathrm{P} 63 \alpha$ or TA-P $63 \alpha$ isoforms of the wt or mutant R379C P63 protein were expressed under the GAL1-10 inducible promoter ( $8 \mathrm{~h}$ at $0.128 \%$ galactose) in two isogenic yeast reporter strains (yLFM-P21-5'; yLFM-PERP) having the luciferase reporter gene under the control of $\mathrm{P} 53$ responsive element present in the promoter region of the P21 and the PERP gene. Presented in the graph is the fold-induction over the empty expression vector (pRS314) of mean luciferase activities normalized to optical density (OD) at $600 \mathrm{~nm}$. Average and standard errors of three biological replicates are plotted. (B) Western blot analysis of different isoforms (TA and $\Delta \mathrm{N}$ ) of $\mathrm{P} 63 \alpha$ proteins revealed that wt and $\mathrm{R} 379 \mathrm{C}$ are expressed at similar level in yeast cells. A specific antibody directed against P63 was used to quantify the P63 isoforms. PGK1 was used as a loading control. The P63/PGK1 ratio is similar within a specific situation (e.g., strain and isoform) for the wt and R379C protein. PGK1, phosphoglycerate kinase 1.

ability cannot be due to a lower amount of mutant TA-P63 $\alpha$ protein compared to the wild-type counterpart, since both proteins were equally expressed (Fig. 3B). These results suggest that, according to our yeast-based P63 functional assay (14), the R379C mutant P63 cannot be immediately classified as a potential pathogenic. In this line it is worth noting that this amino acid is not evolutionarily conserved within P53 family members, suggesting the lack of a key role in the P63 protein functionality; consequently, it is possible to hypothesize that amino acidic substitutions at this position can be well tolerated without markedly altering the transactivation ability of the protein.

\section{Discussion}

NGS approaches of cancer genomes introduce a new scenario also for cutaneous melanomas. Cutaneous melanomas were submitted to NGS for the first time in 2010 (33). Two years later, Hodis et al (34) discovered novel melanoma genes and revealed that the spectrum of mutations provided evidence for a direct mutagenic role of UV light in melanomagenesis. More recently, a distinct sequence of genetic alterations during melanoma progression for different melanoma subtypes was defined $(35,36)$. Benign lesions exclusively harbored BRAF V600E mutation, whereas those categorized as 'intermediate' were enriched for NRAS mutations and additional driver mutations. Biallelic CDKN2A inactivation emerged exclusively in invasive melanomas, while the presence of PTEN and TP53 mutations appears to represent the transition to metastatic tumors (36).

Only recently missense mutations in the TP63 gene were noted to be frequently and specifically found in cutaneous melanoma ( $15 \%)$ (37) in contrast to the previous prevailing notion that TP63 mutations are seldom found in cancer. This observation prompted us to ascertain whether all three members of the $P 53$ family of transcription factors were found mutated in cutaneous melanomas (http://www.cbioportal. org) $(38,39)$. Notably, TP53 and TP63 were frequently mutated in cutaneous melanomas (15.0 and $14.7 \%$, respectively) while the incidence of TP73 mutations (1.5\%) was significantly lower $(\mathrm{p}<0.0001)$.

The TP63 and TP53 mutations found in cutaneous melanomas revealed a UV-recognizable mutation fingerprint, high percentage of GC $>$ AT transitions at PyPy sites, along with a few tandem mutations (Table I). These observations strongly suggest that TP63 and TP53 mutations in cutaneous melanomas are UV-induced. To reinforce this suggestion the TP63 mutations found in cutaneous melanomas were compared with those observed at the germinal level in patients affected by a specific subset of P63-associated disorders (EDs). Since the two sets of mutations should have been induced by different mutagenic events, based on the mutation spectrometry assumption, we anticipated that the two TP63 mutation spectra would be distinguishable. Indeed, they were significantly different. The TP63 mutations in EDs were significantly more frequent at $\mathrm{CpGs}$ sites $(\mathrm{p}<0.0001$; Table II), while those in skin melanomas were significantly more frequent at PyPy sites $(p<0.0001$; Table II). Furthermore, the sequence distribution of independent TP63 base pair substitutions along the nucleotide sequence differed significantly in the two spectra (p<0.0001; Fig. 2). Altogether these results demonstrated that the TP63 mutations observed in cutaneous melanomas are UV-specific, while those observed in EDs are likely of spontaneous origin, with deamination of 5'-mC at $\mathrm{CpG}$ sites being a likely candidate responsible of such events.

Altogether these results suggest that, besides TP53, TP63 mutations, but not TP73, may also play a role in melanomagenesis. However, if this is so, it is expected that the corresponding amino acid substitutions induced by those mutations would show significant functional consequences on the transcriptional activity of the P63 protein. The predictions based on two bioinformatics tools (Mutation Assessor and FATHMM) were rather different. 
While the former discriminated between the effects of specific amino acid substitutions on the P63 protein functionality, the latter predicted that all the mutations were pathogenic (Table III). Therefore, we experimentally determined the functional consequences of the TP63 R379C mutation on the transactivation ability with respect to the wild-type counterpart, since this mutant P63 protein was the most frequent amino acid substitution observed in cutaneous melanomas and the two bioinformatics tools reported different prediction for the same mutation. Using our yeast-based P63 functional assay the R379C protein exhibited a substantial residual activity compared to the wild-type (70-100\%, depending on the yeast reporter strain) (Fig. 3). This result is in keeping with the prediction of the Mutation Assessor and appears to not support a major role of this mutant P63 protein in melanomagenesis, while it is still compatible with the TP63 gene being a recorder of UV exposure. With regard to the TP53 hot spot mutation in melanomas, i.e., R213Stop, it is obligatorily classified as a loss-of-function mutant because the mutation results in a truncated P53 protein that is not able to oligomerize. This observation is in agreement with the predictions of the bioinformatics tools suggesting that TP53 mutations may play a role in melanomagenesis.

While TP53 mutations have been recognized to play a role in the transition to metastatic tumors (36), the indications for the role of TP63 mutations have yet to be elucidated. The fact that $T P 63^{+/-}$mice developed malignant tumors at a higher frequency with respect to the wild-type counterpart, and that the $T P 53^{+/-} \mathrm{TP}^{+/-}$compound mutant mice developed a more severe phenotype (i.e., higher tumor burden and more metastases) compared to the $\mathrm{TP} 53^{+/-}$mice, reveals that TP63 is a tumor/metastasis suppressor gene independent of P53 (15). Thus, TP63 mutations that inactivate the protein function likely contribute to a carcinogenic process. However, besides their relative frequency, TP63 mutations in cutaneous melanomas possibly represent a record of exposure (passenger mutations) rather than driver mutations. This conclusion is supported by additional arguments. Firstly, through an extensive study that considers all type of tumors (48) TP53 is present among the 125 drivers genes recognized, while TP63 is not. Secondly, TP63 and TP53 mutations have a significant tendency towards co-occurrence $(\mathrm{p}<0.001)$ (http:// www.cbioportal.org) in melanomas, a situation that does not support the hypothesis that both mutations may play a major role during melanomagenesis. Thirdly, a possible mechanism through which the mutant P53 protein may facilitate the transition towards metastatic melanomas may be via interference with the antimetastatic function of the wild-type P63. Adorno et al (49) proposed such a mechanism in breast cancer cells, revealing that TGF- $\beta$-dependent cell migration, invasion and metastasis were empowered by the mutant P53 protein and opposed by P63: a mutant P53/P63 protein complex appeared assembled with SMAD proteins which served as an essential platform. Notably, the TGF- $\beta$ /SMAD pathway appears to be active even in melanomas (50). Thus, the mere presence of a mutant P53 protein may be sufficient to antagonize the P63 function without the need to mutate TP63.

It should also be considered that TP53/TP63 mutations may also play different roles according to the phase of cancer development in which they occur. UV-induced TP53/TP63 mutations at PyPy sites are an early event in UV-exposed skin that it is still devoid of any cancer-like phenotype. However, the presence of these mutations with their effects may allow mutated cells to escape apoptosis, thus progressing towards more advanced stages of the carcinogenesis process. In this view, TP53/TP63 may be considered driver mutations of the early carcinogenesis steps. In more advanced cancer stages or in full blown cancer the burden of other mutations prevails, thus giving cancer an aggressive behavior resulting in chemoresistance, metastatisation and decreased survival. Accordingly, TP53/TP63 behave as passenger mutations in these advanced cancer stages.

Two other aspects contribute to maintain the role of TP63 mutations in cutaneous melanomas uncertain. The first is related to a recent study by Matin et al (51) who demonstrated that the two wild-type isoforms of P63, namely TA-P63 and $\triangle \mathrm{N}-\mathrm{P} 63$, play an anti-apoptotic role in melanoma and confer chemoresistance, even in the absence of TP53 mutations. In response to DNA damage, P63 is stabilized in the nuclei and translocates to the mitochondria where it supresses apoptosis through a direct and indirect inhibitory function on P53-dependent pro-apoptotic effectors. In this scenario, the selection of TP63 mutations during melanomagenesis appears to be unnecessary or even deleterious for tumor growth, once again pointing toward a passenger role rather than a driver role for such mutations. Moreover, this observation highlights functions of the P63 protein that are not associated to the key role as nuclear transcription factor. The second aspect is related to the power of the NGS; in fact, while the Sanger sequencing imposed a technical threshold of detection (i.e., a mutation could be identified if present in $20 \%$ of the alleles), NGS can accurately detect mutations at an abundance in the order of $1 \%$ (and even lower). The presence of every mutation identified with NGS facilitate the identification of mutational signatures. However, the biological impact of a mutation that is 1 or $20 \%$ abundant may be different on tumor behavior. In view of understanding which mutations are really important for the carcinogenic process, an interesting way would be to submit to NGS consecutive samples, spaced in time, and to verify what happens to the abundance of specific molecular events in the progression of the tumor. This is relatively easy for some hematological malignancies (e.g., chronic lymphocytic leukemia, CLL); however this appears to be very difficult, or even impossible, for others kind of tumors. In CLL, Rossi et al (52) revealed that TP53 mutations, even in $<1 \%$ of the CLL clone, can be selected upon treatment and therefore should be taken into account when defining therapy protocols.

We conclude that TP63 mutations are frequent in cutaneous melanoma and support UV etiology, however they do not have a clear role in melanomagenesis. The role of TP63 mutations changes according to the stage of melanoma carcinogenesis when they occur being drivers in early stages and passengers in late stages. In the absence of experimental results on gene mutation functionality, bioinformatics tools are often used to predict the effect of a single amino acid substitution on the function of the protein under study. Our results clearly revealed that the experimental data obtained with our yeast-based functional assay may be useful to elucidate the role of mutations on P63 function. 


\section{Acknowledgements}

This study was partially supported by the Italian Association for Cancer Research, AIRC (IG no. 5506 to G.F., IG no. 15460 to P.G.), by Compagnia S. Paolo, Turin, Italy (Project 2012.1590), and by the Ministero della Salute, Fondi 5x1000 2013, Fondi Ricerca Corrente 2016.

\section{References}

1. Lane DP and Crawford LV: T antigen is bound to a host protein in SV40-transformed cells. Nature 278: 261-263, 1979.

2. Kaghad M, Bonnet H, Yang A, Creancier L, Biscan JC, Valent A, Minty A, Chalon P, Lelias JM, Dumont X, et al: Monoallelically expressed gene related to $\mathrm{p} 53$ at $1 \mathrm{p} 36$, a region frequently deleted in neuroblastoma and other human cancers. Cell 90: 809-819, 1997.

3. Yang A, Kaghad M, Wang Y, Gillett E, Fleming MD, Dötsch V, Andrews NC, Caput D and McKeon F: p63, a p53 homolog at 3q27-29, encodes multiple products with transactivating, death-inducing, and dominant-negative activities. Mol Cell 2: 305-316, 1998.

4. Bisio A, Ciribilli Y, Fronza G, Inga A and Monti P: TP53 mutants in the tower of babel of cancer progression. Hum Mutat 35: 689-701, 2014

5. Collavin L, Lunardi A and Del Sal G: p53-family proteins and their regulators: Hubs and spokes in tumor suppression. Cell Death Differ 17: 901-911, 2010.

6. Wei J, Zaika E and Zaika A: p53 family: Role of protein isoforms in human cancer. J Nucleic Acids 2012: 687359, 2012.

7. Malkin D: Li-fraumeni syndrome. Genes Cancer 2: 475-484, 2011.

8. Monti P, Ciribilli Y, Jordan J, Menichini P, Umbach DM, Resnick MA, Luzzatto L, Inga A and Fronza G: Transcriptional functionality of germ line p53 mutants influences cancer phenotype. Clin Cancer Res 13: 3789-3795, 2007.

9. Monti P, Perfumo C, Bisio A, Ciribilli Y, Menichini P, Russo D, Umbach DM, Resnick MA, Inga A and Fronza G: Dominantnegative features of mutant TP53 in germline carriers have limited impact on cancer outcomes. Mol Cancer Res 9: 271-279, 2011.

10. Yang A, Walker N, Bronson R, Kaghad M, Oosterwegel M, Bonnin J, Vagner C, Bonnet H, Dikkes P, Sharpe A, et al: p73-deficient mice have neurological, pheromonal and inflammatory defects but lack spontaneous tumours. Nature 404 99-103, 2000.

11. Mills AA, Zheng B, Wang XJ, Vogel H, Roop DR and Bradley A: p63 is a p53 homologue required for limb and epidermal morphogenesis. Nature 398: 708-713, 1999.

12. Rufini A, Agostini M, Grespi F, Tomasini R, Sayan BS, NiklisonChirou MV, Conforti F, Velletri T, Mastino A, Mak TW, et al: p73 in Cancer. Genes Cancer 2: 491-502, 2011.

13. Rinne T, Brunner HG and van Bokhoven $\mathrm{H}$ : p63-associated disorders. Cell Cycle 6: 262-268, 2007.

14. Monti P, Russo D, Bocciardi R, Foggetti G, Menichini P, Divizia MT, Lerone M, Graziano C, Wischmeijer A, Viadiu H, et al: EEC- and ADULT-associated TP63 mutations exhibit functional heterogeneity toward P63 responsive sequences. Hum Mutat 34: 894-904, 2013.

15. Flores ER, Sengupta S, Miller JB, Newman JJ, Bronson R, Crowley D, Yang A, McKeon F and Jacks T: Tumor predisposition in mice mutant for p63 and p73: Evidence for broader tumor suppressor functions for the p53 family. Cancer Cell 7: 363-373, 2005 .

16. Jacks T, Remington L, Williams BO, Schmitt EM, Halachmi S, Bronson RT and Weinberg RA: Tumor spectrum analysis in p53-mutant mice. Curr Biol 4: 1-7, 1994.

17. Vogelstein B and Kinzler KW: Carcinogens leave fingerprints. Nature 355: 209-210, 1992.

18. Harris CC: p53: At the crossroads of molecular carcinogenesis and risk assessment. Science 262: 1980-1981, 1993.

19. Fronza G, Inga A, Monti P, Scott G, Campomenosi P, Menichini P, Ottaggio L, Viaggi S, Burns PA, Gold B, et al: The yeast p53 functional assay: A new tool for molecular epidemiology. Hopes and facts. Mutat Res 462: 293-301, 2000.

20. Leroy B, Fournier JL, Ishioka C, Monti P, Inga A, Fronza G and Soussi T: The TP53 website: An integrative resource centre for the TP53 mutation database and TP53 mutant analysis. Nucleic Acids Res 41 (D1): D962-D969, 2013.
21. Greenblatt MS, Bennett WP, Hollstein M and Harris CC: Mutations in the p53 tumor suppressor gene: Clues to cancer etiology and molecular pathogenesis. Cancer Res 54: 48554878, 1994.

22. International Agency for Research on Cancer (IARC): IARC Monographs on the Evaluation of Carcinogenic Risk of Chemicals to Humans: Solar and Ultraviolet Radiation. Vol. 55 IARC, Lyon, France, 1992 (http://monographs.iarc.fr/ENG/ Monographs/vol55)

23. Clingen PH, Arlett CF, Roza L, Mori T, Nikaido O and Green MH: Induction of cyclobutane pyrimidine dimers, pyrimidine(6-4)pyrimidone photoproducts, and Dewar valence isomers by natural sunlight in normal human mononuclear cells. Cancer Res 55: 2245-2248, 1995.

24. Cadet J, Sage E and Douki T: Ultraviolet radiation-mediated damage to cellular DNA. Mutat Res 571: 3-17, 2005.

25. Vogelstein B and Kinzler KW: Cancer genes and the pathways they control. Nat Med 10: 789-799, 2004.

26. Parmigiani G, Boca S, Lin J, Kinzler KW, Velculescu V and Vogelstein B: Design and analysis issues in genome-wide somatic mutation studies of cancer. Genomics 93: 17-21, 2009.

27. Carter H, Chen S, Isik L, Tyekucheva S, Velculescu VE, Kinzler KW, Vogelstein B and Karchin R: Cancer-specific highthroughput annotation of somatic mutations: Computational prediction of driver missense mutations. Cancer Res 69: 6660-6667, 2009.

28. Youn A and Simon R: Identifying cancer driver genes in tumor genome sequencing studies. Bioinformatics 27: 175-181, 2011.

29. Vogelstein B and Kinzler KW: The path to cancer - three strikes and you're out. N Engl J Med 373: 1895-1898, 2015.

30. Tomasetti C, Marchionni L, Nowak MA, Parmigiani G and Vogelstein B: Only three driver gene mutations are required for the development of lung and colorectal cancers. Proc Natl Acad Sci USA 112: 118-123, 2015.

31. Miller AJ and Mihm MC Jr: Melanoma. N Engl J Med 355: 51-65, 2006.

32. Marks R: Epidemiology of melanoma. Clin Exp Dermatol 25: 459-463, 2000

33. Pleasance ED, Cheetham RK, Stephens PJ, McBride DJ, Humphray SJ, Greenman CD, Varela I, Lin ML, Ordóñez GR, Bignell GR, et al: A comprehensive catalogue of somatic mutations from a human cancer genome. Nature 463: 191-196, 2010.

34. Hodis E, Watson IR, Kryukov GV, Arold ST, Imielinski M, Theurillat JP, Nickerson E, Auclair D, Li L, Place C, et al: A landscape of driver mutations in melanoma. Cell 150: 251-263, 2012.

35. Shain AH, Yeh I, Kovalyshyn I, Sriharan A, Talevich E, Gagnon A, Dummer R, North J, Pincus L, Ruben B, et al: The genetic evolution of melanoma from precursor lesions. N Engl J Med 373: 1926-1936, 2015.

36. Shain AH and Bastian BC: From melanocytes to melanomas. Nat Rev Cancer 16: 345-358, 2016.

37. Romano RA and Sinha S: Family matters: Sibling rivalry and bonding between p53 and p63 in cancer. Exp Dermatol 23: 238-239, 2014.

38. Gao J, Aksoy BA, Dogrusoz U, Dresdner G, Gross B, Sumer SO, Sun Y, Jacobsen A, Sinha R, Larsson E, et al: Integrative analysis of complex cancer genomics and clinical profiles using the cBioPortal. Sci Signal 6: pl1, 2013.

39. Cerami E, Gao J, Dogrusoz U, Gross BE, Sumer SO, Aksoy BA, Jacobsen A, Byrne CJ, Heuer ML, Larsson E, et al: The cBio cancer genomics portal: An open platform for exploring multidimensional cancer genomics data. Cancer Discov 2: 401-404, 2012.

40. Berger MF, Hodis E, Heffernan TP, Deribe YL, Lawrence MS, Protopopov A, Ivanova E, Watson IR, Nickerson E, Ghosh P, et al: Melanoma genome sequencing reveals frequent PREX2 mutations. Nature 485: 502-506, 2012.

41. Krauthammer M, Kong Y, Ha BH, Evans P, Bacchiocchi A, McCusker JP, Cheng E, Davis MJ, Goh G, Choi M, et al: Exome sequencing identifies recurrent somatic RAC1 mutations in melanoma. Nat Genet 44: 1006-1014, 2012.

42. Rinne T, Bolat E, Meijer R, Scheffer H and van Bokhoven H: Spectrum of $\mathrm{p} 63$ mutations in a selected patient cohort affected with ankyloblepharon-ectodermal defects-cleft lip/palate syndrome (AEC). Am J Med Genet A 149A: 1948-1951, 2009.

43. Clements SE, Techanukul T, Coman D, Mellerio JE and McGrath JA: Molecular basis of EEC (ectrodactyly, ectodermal dysplasia, clefting) syndrome: Five new mutations in the DNA-binding domain of the TP63 gene and genotype-phenotype correlation. Br J Dermatol 162: 201-207, 2010. 
44. Adams WT and Skopek TR: Statistical test for the comparison of samples from mutational spectra. J Mol Biol 194: 391-396, 1987.

45. CarielloNF,Piegorsch WW,Adams WT andSkopekTR:Computer program for the analysis of mutational spectra: Application to p53 mutations. Carcinogenesis 15: 2281-2285, 1994.

46. Andreotti V, Ciribilli Y, Monti P, Bisio A, Lion M, Jordan J, Fronza G, Menichini P, Resnick MA and Inga A: p53 transactivation and the impact of mutations, cofactors and small molecules using a simplified yeast-based screening system. PLoS One 6: e20643, 2011.

47. Kushnirov VV: Rapid and reliable protein extraction from yeast. Yeast 16: 857-860, 2000.

48. Vogelstein B, Papadopoulos N, Velculescu VE, Zhou S, Diaz LA Jr and Kinzler KW: Cancer genome landscapes. Science 339: 1546-1558, 2013.
49. Adorno M, Cordenonsi M, Montagner M, Dupont S, Wong C, Hann B, Solari A, Bobisse S, Rondina MB, Guzzardo V, et al: A Mutant-p53/Smad complex opposes p63 to empower TGFbeta-induced metastasis. Cell 137: 87-98, 2009.

50. Perrot CY, Javelaud D and Mauviel A: Insights into the transforming growth factor- $\beta$ signaling pathway in cutaneous melanoma. Ann Dermatol 25: 135-144, 2013.

51. Matin RN, Chikh A, Chong SL, Mesher D, Graf M, Sanza' P, Senatore V, Scatolini M, Moretti F, Leigh IM, et al: p63 is an alternative p53 repressor in melanoma that confers chemoresistance and a poor prognosis. J Exp Med 210: 581-603, 2013.

52. Rossi D, Khiabanian H, Spina V, Ciardullo C, Bruscaggin A, Famà R, Rasi S, Monti S, Deambrogi C, De Paoli L, et al: Clinical impact of small TP53 mutated subclones in chronic lymphocytic leukemia. Blood 123: 2139-2147, 2014. 